\title{
Palmeras usadas por los indígenas Asháninkas en la Amazonía Peruana
}

\author{
Palms used by Ashaninka indigenous people in Peruvian Amazon
}

\author{
Joanna Sosnowska', Damaso Ramirez² y Betty Millán²
}

\begin{abstract}
1 W. Szafer Instituto de Botánica, Academia Polaca de Ciencias, ul. Lubicz 46, 31-512 Cracovia, Polonia.

Email Joanna Sosnowska: j.sosnowska@botany.pl

2 Museo de Historia Natural, Universidad Nacional Mayor de San Marcos, Avda. Arenales 1256 Jesús María, Lima 14, Perú.

Email Damaso Ramirez: wilsonXVIII@gmail.com,

Email Betty Millán: bmillans@ unmsm.edu.pe, bmillans@gmail.
\end{abstract} com

\section{Presentado: $\quad$ 12/02/2010}

$23 / 07 / 2010$

\begin{abstract}
Resumen
El presente artículo muestra el conocimiento e importancia de las palmeras en la vida de los nativos Asháninkas. Presentamos una descripción cualitativa y cuantitativa de 32 entrevistas, obtenidos durante la visita a siete comunidades nativas ubicadas en los márgenes de los ríos Perené y Tambo en el departamento Junín, Perú Registramos 15 especies de palmeras usadas por los Asháninkas, agrupadas bajo cinco categorías de uso: alimenticio, construcción, herramienta, ornamental y medicinal. Las especies con usos más amplios son: Attalea phalerata, Bactris gasipaes, Oenocarpus bataua y Socratea exorhiza. Las partes de las palmeras más utilizadas son los frutos, principalmente gracias a su valor comestible. La cercanía de las comunidades Asháninkas del valle del Perené a ciudades, influirían en un cambio en el tipo de vida tradicional, donde las palmeras son los más importantes recursos naturales utilizados por ellos. Sin embargo, en las comunidades del valle Tambo la vida tradicional, el conocimiento y practica en el uso de las palmeras esta aún vital.
\end{abstract}

Palabras clave: Arecaceae, Asháninka, etnobotánica, Perú.

\section{Abstract}

In this paper we present traditional knowledge and importance of palms in Ashaninka people's life. Qualitative and quantitative description is based on 32 interviews obtained during visits in seven native communities situated near by Perene and Tambo rivers in Junín department of Peru. We registered 15 species of palms used by Ashaninka people; those were classified in five categories by use: food, construction, tools, ornaments and medicaments. Species with the most diverse uses were Attalea phalerata, Bactris gasipaes, Oenocarpus bataua and Socratea exorhiza. The most useful palm parts are fruits used mainly as a food. The proximity of Asháninkas communities from Perené Valley to the cities would produce a change in the traditional way of life, where palm trees are the most important natural resources used by them. However, the traditional life, knowledge and practice in using of palms are still alive in communities of Tambo River.

Keywords: Arecaceae, Ashaninka, ethnobotany, Peru.

\section{Introducción}

Los Asháninkas son uno de los pueblos nativos más numerosos de la Amazonía peruana. Mucho se ha escrito sobre su cultura, organización social y violencia política en su territorio (Varese 2006, Weiss 2005). En este artículo intentamos mostrar el conocimiento e importancia de las palmeras en su vida.

Tradicionalmente, los Ashaninkas vivían como familias dispersas en el monte, orgullosos de su autonomía, libertad y resistencia a los diferentes cambios desde la época de la conquista española (Weiss 2005). La conformación de comunidades nativas fue impuesta por el Estado peruano en los años de 1970, siguiendo el modelo andino (Espinosa 1996). Los políticos de ese entonces y los de ahora, no solo ignoran las características específicas de la organización social en la selva, sino también las tecnologías del manejo del bosque desarrolladas por los Ashánincas, para mantener el frágil equilibrio ecológico de la Amazonía.

Hoy en día la cuenca del río Perené, poblado por los Asháninkas y grandes poblaciones de colonos, es una fuente abundante de productos agrícolas. Plantaciones de café, cítricos, plátanos y otros frutos dominan el paisaje del valle Perené. Este panorama es muy diferente en comparación con el valle Tambo que fue aislado por "Sendero Luminoso" en la época de la violencia y donde además el acceso es más difícil. Hoy en día los Asháninkas aún dependen de los recursos que le brinda la selva para su subsistencia, dentro de los cuales las palmeras forman parte importante como alimento (Mauritia flexuosa, Oenocarpus bataua, Bactris gasipaes), fibras (Astrocaryum chambira) o construcción (Socratea exorrhiza, Iriartea deltoidea). Todos estos productos forman parte de la vida cotidiana de los nativos amazónicos.
En el presente artículo damos a conocer los resultados del estudio etnobotanico sobre las palmeras, obtenidos durante la expedición realizada a comunidades Asháninkas del Departamento de Junín, en la Amazonía peruana.

\section{Área del estudio}

Entre octubre y diciembre de 2008, se visitaron siete comunidades nativas Asháninka. Cinco ubicadas en la cuenca del río Perené: Marankiari, Bajo Aldea, Zotani, Pitocuna, Impitato Cascada, y dos ubicadas en la cuenca del río Tambo: Puerto Ocopa y Savareni (Tabla 1) en el Departamento de Junín (Fig.1).

\section{Material y métodos}

Se realizaron colectas intensivas de las palmeras utilizadas, con la ayuda de miembros de cada comunidad nativa evaluada, los cuales además fueron nuestros guías. Se realizaron observaciones y entrevistas acerca de los usos de las palmeras. La información fue recolectada realizando entrevistas semi-estructuradas (Alexiades 1996) a 30 pobladores indígenas Asháninkas y dos personas de otro origen (uno Asháninka/Yánesha y otro Piro). A los entrevistados se les mostraron las palmeras que se habían colectado en la zona.

Entre las 32 personas entrevistadas, la gran mayoría hablan en su idioma nativo (Asháninka), por lo cual registramos los nombres de las palmeras en su idioma nativo y los nombres usados en español. Las muestras fueron determinadas de acuerdo a Henderson et al. (1995) y Kahn \& Millán (1992). Los nombres científicos de las especies siguen la clasificación presentada por Dransfield et al. (2008) y Govaerts y Dransfield (2005).

La colección completa fue depositada en el Herbario del W. Szafer Instituto de Botánica (KRAM) de la Academia Polaca de 
Figura 1. Ubicación de las siete comunidades Asháninka del presente estudio, Departamento Junín, Perú.

Tabla 1. Comunidades Asháninka en el Departamento de Junín en la Amazonía de Perú, visitados en 2008 con el propósito de estudiar usos de las palmeras (Arecaceae), indicando en cual río están localizados, las coordenadas y altitud sobre el nivel del mar medido con GPS.

\begin{tabular}{cccc}
\hline Río & Comunidad & Coordenadas & Altitud (m) \\
\hline Peréne & Bajo Marankiari & $10^{\circ} 56^{\prime} \mathrm{S}, 75^{\circ} 11^{\prime} \mathrm{W}$ & 650 \\
Peréne & Bajo Aldea & $10^{\circ} 53^{\prime} \mathrm{S}, 74^{\circ} 54^{\prime} \mathrm{W}$ & 560 \\
Peréne & Zotani & $1^{\circ} 51^{\prime} \mathrm{S}, 74^{\circ} 55^{\prime} \mathrm{W}$ & 850 \\
Peréne & Pitocuna & $11^{\circ} 00^{\prime} \mathrm{S}, 74^{\circ} 39^{\prime} \mathrm{W}$ & 470 \\
Peréne & Impitato Cascada & $11^{\circ} 00^{\prime} \mathrm{S}, 74^{\circ} 40^{\prime} \mathrm{W}$ & 460 \\
Tambo & Puerto Ocopa & $11^{\circ} 08^{\prime} \mathrm{S} 74^{\circ} 10^{\prime} \mathrm{W}$ & 370 \\
Tambo & Savareni & $1^{\circ} 13^{\prime} \mathrm{S}, 73^{\circ} 41^{\prime} \mathrm{W}$ & 270 \\
\hline
\end{tabular}

Ciencias en Cracovia y en el Herbario San Marcos (USM) del Museo de Historia Natural de la Universidad Nacional Mayor de San Marcos en Lima, Perú.

\section{Resultados}

Se encontraron un total de 15 especies de palmeras usadas por los pobladores de las 7 comunidades nativas visitadas. La especie Cocos nucifera (coco)' era considerado por los Asháninkas como 'no natural' y no fue incluido en este estudio.

A continuación, presentamos una descripción cualitativa y cuantitativa de nuestras observaciones de las diferentes formas en las cuales las palmeras son utilizadas en el área de estudio. Las descripciones están organizadas alfabéticamente por especie y bajo cinco categorías de uso: alimenticio, construcción, herramienta, ornamental y medicinal. También incluimos los nombres en español y Asháninka. Las especies registradas cuentan con un espécimen de herbario, los números de colección se encuentran citados bajo la denominación de "muestra", representando las colecciones realizadas por J. Sosnowska y D. Ramirez y depositadas en los herbarios KRAM y USM.

\section{Astrocaryum perangustatum F.Kahn \& B.Millán}

Asháninka: tiroti; Español: huikungo

Uso alimenticio.- Los frutos son comestibles; el palmito es extraído para su consumo; las inflorescencias son recolectadas y consumidas cocidas; las larvas de coleóptero (suris) que se desarrollan en los troncos caídos son recolectados y consumidas crudos o cocidos.

Uso en construcción.- El tronco es utilizado como postes de las casas y las hojas suelen ser utilizadas para el techado de las viviendas.

Uso como herramienta. - Las semillas se usan para elaborar artesanías; las espinas se usan como agujas y puntas de flechas; las hojas jóvenes, son utilizados en la fabricación de abanicos, sopladores y esteras, también para la fabricación de canastas para guardar yuca o algodón; las fibras del raquis son utilizadas para hacer escobas.

Uso medicinal.- Las raíces son utilizadas en la preparación de un extracto contra la hepatitis.

Muestra: JS -30 .

\section{Attalea phalerata Mart. ex Spreng.}

Asháninka: tsiaro; Español: shapaja

Uso alimenticio.- Los frutos y sus aceite son comestibles; las semillas son colectadas para preparar mantequilla; el palmito es extraído para consumo; las larvas de coleóptero (suris) son cosechadas de los troncos en descomposición y luego consumidos crudos o cocidos.

Uso en construcción. - El tronco se utiliza en construcción de postes; las hojas en el techado de las viviendas. 
Uso como herramienta. - Los frutos se usan en la elaboración de artesanías; las hojas son usadas para la fabricación de abanicos, sopladores de fuego, esteras, paneras y canastas; las fibras del raquis son utilizadas para hacer escobas; la bractea se usa como fósforos, ya que se mantiene encendida por un tiempo; la inflorescencia es usada por los niños como juguete.

Uso ornamental.- Los frutos y flores son usados como adorno.

Uso medicinal.- El aceite extraído del fruto se usa como loción para mejorar la piel de la cara; las raíces son utilizadas para la preparación de un extracto contra la hepatitis, es más fuerte que el extracto de tiroti (Astrocaryum perangustatum).

Muestra: JS - 29.

\section{Bactris gasipaes Kunth var. gasipaes}

\section{Asháninka: kiri; Español: pijuayo}

Uso alimenticio.- Los frutos maduros son utilizados para la preparación de bebidas (masato) y la extracción de aceite; las larvas de coleópteros (suris) son recolectados de los troncos en descomposición y consumidas crudas o cocidas.

Uso en construcción.- Los clavos preparados del tronco se usan en la construcción de las balsas.

Uso como herramienta.- Los troncos son utilizados en la fabricación de flechas y arcos. También se usan los "palos muy delgados" para hilar algodón. Las hojas pueden servir para fabricar esteras y abanicos.

Uso medicinal.- La raíz es utilizada para la preparación de un extracto usado contra la infección urinaria.

Muestra: JS - 3 .

\section{Bactris sp.}

Asháninka: ashanki; Español: nejilla

Uso alimenticio.- Las semillas son consumidas como caramelos.

Uso como herramienta.- Las semillas son utilizados para elaborar collares y del peciolo de la hoja se fabrican flechas

Uso medicinal.- La raíz es utilizada para la preparación de un extracto usado contra la calvicie. Las espinas son utilizadas para retirar otras espinas incrustadas en las personas.

Muestra: JS - 10 .

\section{Chamaedorea angustisecta Burret}

\section{Asháninka: shikashika}

Uso en construcción. - Las hojas suelen ser utilizadas para la construcción del techado de las viviendas.

Uso como herramienta.- Las semillas son usadas para la elaboración de collares; la flor es utilizada como adorno; las hojas son utilizadas en la cocina, para envolver el pescado y para hacer sopladores de fuego; los troncos son utilizados en la fabricación de arcos y flechas para niños, también se extraen "palos delgados" para hilar algodón; el tronco con raíces es utilizado como escoba

Uso ornamental.- La flor es utilizada para elaborar perfumes.

Uso medicinal.- La pepa del fruto es utilizado como compresa para las dolencias dermatológicas.

Muestra: JS - 6 .

\section{Elaeis guineensis Jacq.}

Asháninka: datil.

Uso alimenticio.- Los frutos son comestibles.

Uso en construcción.- Las hojas sirven para la fabricación del techo de las viviendas.

Muestra: JS - 41 .

\section{Euterpe precatoria Mart.}

Asháninka: tsirentsi; Español: huasai

Uso alimenticio. - Los frutos de tsirentsi son utilizados para preparar un refresco o es mezclado con yuca para preparar masato (tradicional bebida alcohólica de la Amazonía); el palmito es extraído para consumo; las larvas de coleóptero (suris) que se desarrollan en los troncos caídos son recolectados y consumidos, crudos o cocidos.

Uso en construcción. - Ocasionalmente las hojas son utilizadas para la fabricación del techo de las casas y el tronco para la fabricación de cercos (en deficiencia de otro material mejor);

Uso como herramienta. - Las semillas se usan para elaborar collares; las hojas son utilizadas para la fabricación de esteras, sopladores, abanicos y canastas

Uso medicinal.- Las raíces son utilizadas para la preparación de un extracto usado contra el dolor de espalda, huesos, infección de útero, riñones y para hacer crecer el pelo de los niños.

Muestra: JS - 39 .

\section{Geonoma interrupta (Ruiz \& Pav.) Mart.}

Asháninka: kapashi; Español: palmichi

Uso alimenticio.- Los frutos son comestibles

Uso en construcción.- Las hojas son utilizadas para la fabricación de los techos de viviendas; los troncos son utilizados como cerca.

Uso como herramienta.- Los frutos se usan para elaborar artesanías; el tronco para fabricar flechas.

Uso medicinal.- La hoja y la raíz se usan en el baño de los niños con el fin de fortalecer su salud.

Muestra: JS -7 .

\section{Iriartea deltoidea Ruiz \& Pav.}

Asháninka: camona; Español: pona

Uso alimenticio.- Los frutos verdes son comestibles sola- 
mente cocidos; las larvas de coleópteros (suris) que desarrollan en los troncos caídos son recolectados y consumidos.

Uso en construcción. - Las hojas son utilizadas para la fabricación de un techado temporal; la madera obtenida del tronco, es utilizada para los pisos y las paredes de las viviendas, los troncos duros son utilizados para postes, tarima, cama y cerca.

Uso como herramienta. - Los frutos se usan para elaborar artesanías; las hojas se usan para fabricar esteras.

Uso medicinal.- El palmito es consumido para mejorar el hígado; la raíz se aplasta y se aplica directo al pie inflamado; la flor es usada, durante el baño de los niños, para la curación del susto.

Muestra: JS - 8.

\section{Mauritiella sp.}

Español: aguajillo

Uso alimenticio.- Los frutos son comestibles.

Muestra: sin muestra colectada.

\section{Mauritia flexuosa L.f.}

\section{Asháninka: toniro; Español: aguaje}

Uso alimenticio.- Los frutos son comestibles y utilizados para la elaboración de refresco aguajina; las larvas de coleópteros (suris) que se desarrollan en los troncos caídos son recolectados y consumidos.

Uso en construcción.- Las hojas son utilizadas en la construcción del techado temporal de las casas.

Uso como herramienta.- Los frutos y las hojas se usan para elaborar artesanías

Uso ornamental.- Los frutos son usados como adornos ornamentales.

Uso medicinal.- Los frutos tienen propiedades afrodisíacos.

Muestra: JS - 12 .

\section{Oenocarpus bataua Mart.}

\section{Asháninka: shaki; Español: hungurawi}

Uso alimenticio.-Los frutos son comestibles y utilizados en la preparación de refresco, chicha, masato, chocolate y aceite; el palmito es extraído para su consumo; las larvas de coleóptero (suris) que se desarrollan en los troncos caídos son recolectados y consumidas.

Uso en construcción. - Las hojas suelen ser utilizadas para la fabricación del techo de las viviendas.

Uso como herramienta. - - Los frutos se usan para elaborar artesanías, las semillas para elaborar botones; las hojas jóvenes se usan para la fabricación de artesanías, abanicos, canastas, paneras y esteras; la hoja o inflorescencia se usa como escoba.

Uso medicinal.- Los frutos se usan contra la diabetes y cáncer; el palmito es consumido para mejorar el hígado; la raíz es utilizada para preparar una infusión contra la diabetes y paludismo; la raíz también es usada para teñir el cabello.

Muestra: JS -9 .

\section{Oenocarpus mapora H.Karst.}

Asháninka: chorinaki; Español: sinamillo

Uso alimenticio.-De los frutos se prepara refresco; el palmito es comestible.

Uso en construcción. - Las hojas son utilizadas para el techado de las viviendas; los troncos son utilizados como postes.

Uso como herramienta. - Las hojas son utilizadas para la fabricación de esteras y abanicos; de la madera del tronco se fabrican flechas.

Muestra: JS - 40 .

\section{Phytelephas macrocarpa Ruiz \& Pav.}

\section{Asháninka: humiro; Espańol: yarina}

Uso alimenticio.- Las semillas recién formadas son comestibles y se sirve en reuniones para degustar; las flores son importantes para obtener miel de las abejas

Uso en construcción. - Las hojas son utilizadas para la fabricación del techado de las viviendas.

Uso como herramienta.- - Las semillas maduras se usan ocasionalmente para elaborar artesanías pero son un material muy duro y difícil de trabajar; del fruto se fabrican vajillas, las hojas se usan para elaborar canastas temporales para la pesca; los peciolos de las hojas verdes son utilizados para la fabricación de canastas; de las hojas también se fabrican abanicos; la inflorescencia es usada por los niños como juguete.

Uso ornamental. - Las mujeres usan las hojas y flores de humiro como adorno; las hojas son utilizadas para la construcción de la casa ritual, donde se alberga a las mujeres en su primera menstruación.

Uso medicinal.- El liquido de los frutos es usado para curar las infecciones de los ojos, estomago rińones y susto; la raíz es usado como cicatrizante; la flor es utilizada para prepara un extracto contra la esterilidad masculina.

Muestra: JS - 2 .

\section{Socratea exorrhiza (Mart.) H.Wendl.}

Asháninka: tsentero; Español: kashapona

Uso alimenticio.- El palmito es comestible.

Uso en construcción.- Las hojas son utilizadas para el techado temporal de las casas; los troncos son usados en la construcción de paredes, piso, cerca y cama.

Uso como herramienta.- La pepa de los frutos se usa para colorear la kushma (la ropa tradicional); las hojas son usadas para la fabricación de corrales de aves; la raíz es usada como utensilio de rallador en la cocina.

Uso medicinal.- La raíz se usa para fortalecer físicamente a los varones y en el baño de los nińos para alejar el "mal espiritu". 


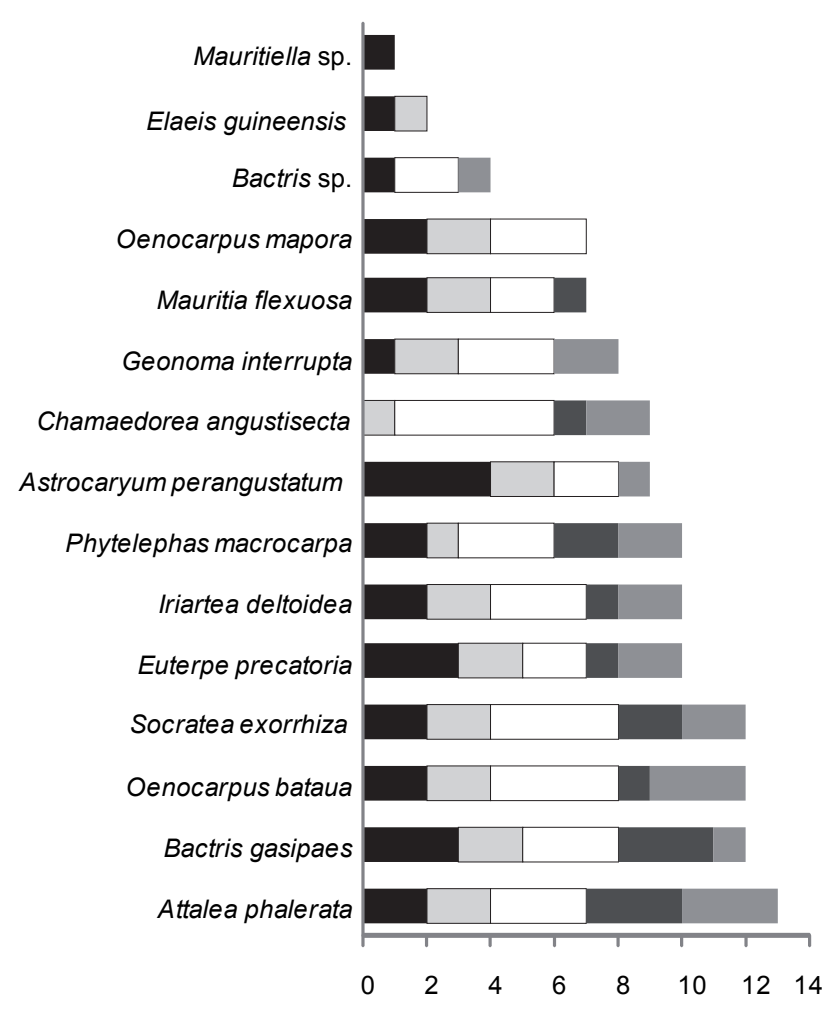

Número de partes por categorías de uso

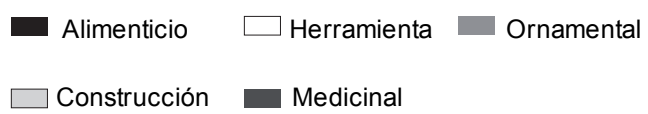

Figura 2. Las palmeras con usos mas amplios - numero de partes usadas de la especie por categorías de uso.

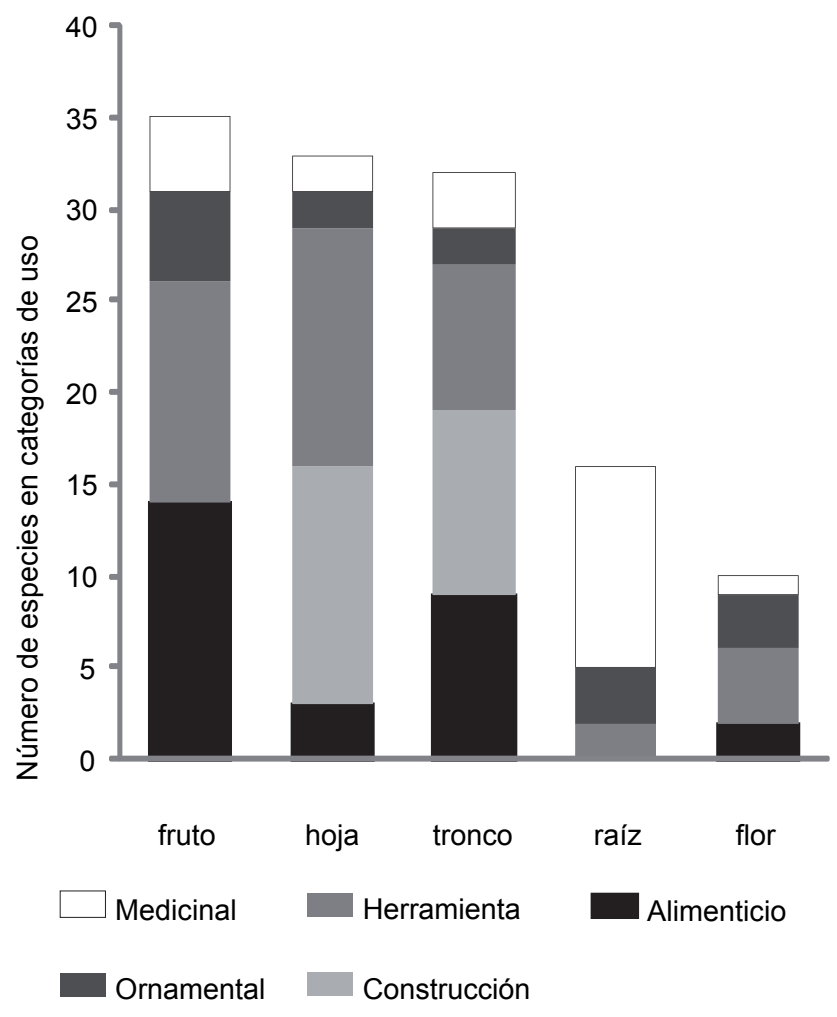

Figura 3. Importancia de las partes usadas de las especies de palmeras por categoría de uso.
Después de bañar, se pasa entre las raíces y se come plátano muy maduro para incitar el vomito.

Muestra: JS - 36 .

Las especies con mayor cantidad de usos fueron: Attalea phalerata, Bactris gasipaes, Oenocarpus batana y Socratea exorrhiza. La importancia es descrita como el número de partes de la palmera en cada categoría de uso. Los usos de las palmeras son muy diversos. Siete especies tienen aplicaciones en todas las categorías (Fig. 2).

Las partes de las palmeras más utilizadas son los frutos, principalmente por su valor comestible. Solamente las hojas y troncos son útiles en construcción, principalmente para la fabricación de techos y postes de casa. La gran importancia de los troncos en la categoría de uso alimenticio resulta porque son hábitat de las larvas de coleópteros (suris), que se cosechan de los troncos caídos. Entre los usos medicinales más importantes de las palmeras, resaltan las raíces (Fig. 3).

\section{Conclusión}

Encontramos una gran variación de uso de las palmeras entre las comunidades Ashaninka estudiadas. También se observa variación en los informantes, en cuáles y cuántos usos un conocía; estas variaciones dependieron de la edad y sexo de cada informante. De hecho, las diferencias en conocimiento de usos de las palmeras entre las comunidades fueron mayores que las encontradas entre edad y sexo de los informantes. Las comunidades del valle Perené están mas cerca de la ciudades que las comunidades del valle Tambo. Hoy en día la cuenca del rio Perené está poblada por colonos, y expone a los nativos a un contacto intensivo con la cultura occidental, que resulta en un cambio del tipo de vida tradicional, donde las palmeras son los más importantes recursos naturales. La vida tradicional, conocimiento y práctica en el uso de palmeras esta aún vital en comunidades del valle Tambo.

\section{Kantantsi orijaniki}

(Resumen en idioma Asháninka)

Oka sankenarentsi oinijantiro jaoka okantasanotari ora shaashi ashaninkaiteki.Okantakoti kenketsarentsi okaratake 32 sampitantsi cokarati oshirinkapaka 7 nanpitsi saikajetatsiri ashaninka perenesati aisati tamposati aka satipoki Satipo, Junín, Perú. Osankinatakotaka ocarati 15 shaaniro palmeras, yobayetari aisati yantantaro ibanko, ichakopite, ipankitiro aisati isayetaro ashaninkaite. Ora yantasanoyetiri ojita tsiaro, kiri, shaki aisati tsentero. Ora yaasanoyetiri shaaniro palmera oitsoki orointa yoyetari. Nanpitsipee timayetatsiri pereneki otimi kempeji antyiaroite nanpitsi isaikinta birakocha, iro kantakairo ipiakojetantakaroki yantayetiri acharineite, itimati oshekiti oshiyoki shaaniro palmeras. Anta tampoki irosati imatiro yantiro antantsi aisati iyotantsi yantirini acharineite.

Iropero ñaantsi: Arecaceae, Asháninka, etnobotánica, Perú.

\section{Agradecimientos}

Agradecemos a la gente Asháninka por compartir su conocimiento de palmeras con nosotros y a las organizaciones CECONSEC y CART gracias a las cuales nuestra investigación fue possible. Gracias a José David Rivera Shiaretti por la traducción del resumen a idioma Asháninka y Miroslaw Rajter por su ayuda en la preparación de los documentos indispensable para conducir trabajo en el campo. Esta publicación se ha realizado gracias a 
la Autorización No 139-2008-INRENA-IFFS-DCB otorgada por el Instituto Nacional de Recursos Naturales del Ministerio de Agricultura de la República del Perú. Se agradece el apoyo económico del Ministerio de Ciencias y Educación Superior del Polonia (N N305 022036).

\section{Literatura citada}

Alexiades M. N. 1996. Collecting ethnobotanical data: an introduction to basic concepts and techniques. Pp. 53-94. En: M. N. Alexiades (ed.) Selected guidelines for ethnobotanical research: a field manual. Advances in economic botany 10. New York Botanical Garden, Bronx, N.Y.

Dransfield, J., N.W. Uhl, C.M. Asmussen, et al. 2008. Genera Palmarum. The Evolution and Classification of Palms. The Board of Trustees of Royal Botanic Gardens, Kew.
Espinosa O. 1996. El pueblo Asháninka y su lucha por la ciudadanía en un país pluricultural. Pp. 77-132. En: Derechos humanos y pueblos indígenas de la amazonía peruana. Lima: APEP - CAAAP.

Govaerts R. \& J. Dransfield. 2005. World Checklist of Palms. The Board of Trustees of Royal Botanic Gardens, Kew.

Henderson, A., G. Galeano, R. Bernal. 1995. Field guide to the palms of the Americas. Princeton University Press, New Jersey.

Kahn, F. \& B. Millán. 1992. Astrocaryum (Palmae) in Amazonia. A preliminary treatment. Bull. Inst. Fr. Ét. And. 21(2): 459-531.

Varese S. 2006. La sal de los cerros. Resistencia y utopia en la amazonía peruana. Fondo Editorial del Congreso del Perú, Lima.

Weiss G. 2005. Campa ribereños. Pp. 1-74. En: Santos F. \& F. Barclay (eds.) Guía Etnográfica de la Alta Amazonía, vol 5. STRI - IFEA, Lima. 